\title{
Survey on Segmentation Methods for Locating Masses in a Mammogram Image
}

\author{
Prof. Samir Kumar Bandyopadhyay \\ Dept. of Computer Sc. \& Engg, University of Calcutta \\ 92 A.P.C. Road, Kolkata - 700009, India
}

\begin{abstract}
A digital mammogram generally detects varying degrees of breast cancer such as clustered microcalcifications, speculated lesions, circumscribed masses, ill-defined masses, and architectural distortions. Many methods of analysing digital mammograms have been recently examined and yielded varied success.

Common techniques from the field of image processing have been applied to digital mammograms in an effort to locate signs of cancer sooner and more precisely than previously possible. Research suggests that computerized techniques applied/utilized by radiologists will be highly successful in analysing digital mammograms. Computerized systems that draw attention to areas of suspicion, otherwise less noticeable to radiologists have the potential to greatly increase early detection.
\end{abstract}

Previously, Algorithms that effectively segment mammogram images into major sub-components and also meets the goals of efficiency and generality has been lacking. This paper reviews some methods of mammogram segmentation process for detection of masses in breast.

\section{INTRODUCTION}

Cancer is a group of many related diseases that begin in cells, the body's basic unit of life. The body is made up of many types of cells. Normally, cells grow and divide to produce more cells only when the body needs them. This orderly process helps keep the body healthy. Sometimes, however, cells keep dividing when new cells are not needed. These extra cells form a mass of tissue, called a growth or tumor.

Tumors can be benign or malignant. Cells from benign tumor do not spread to other parts of the body and can be removed if necessary, although benign breast tumors are not life-threatening. Malignant tumors can invade and damage nearby tissues and organs, and spread to other parts of the body, a process called metastasis.

There are several techniques for locating masses in mammogram images. They function with varying levels of success, and many of them utilize a form of template matching to compare parts of an image with a selected model of a suspicious mass.

Others construct feature vectors and employ a pre-trained classifier to choose malignant areas. A significant shortcoming of many screening algorithms is that they are tested on limited numbers of images. This is usually due to the amount of time needed to evaluate, record, and compare the results of the algorithms. Much of this work must be done manually, including comparing computer results to pathology.

Many consider early detection the key to surviving breast cancer. The most effective method of screening is mammography, which can detect a tumor up to two years before a lump can be felt.

The specific causes of breast cancer are not known. However, there are some risk factors that may affect one's chances of developing breast cancer. They include early or late onset of menopause, family history of breast cancer, hormone replacement therapy, and certain dietary factors.

For treatment purposes, breast cancers have been divided into five stages:

I. Stage zero describes cancer cells that are non-invasive but pose a long-term risk of becoming invasive.

II. Stage One (I) describes tumors no more than 2 centimeters across that have not spread beyond the breast.

III. In stage Two (II), the tumor is about 2 centimeters but has spread to the lymph nodes under the arm, or the tumor is about 5 centimeters but has not spread to the lymph nodes under the arm.

IV. Stage Three (III) cancers are more than 5 centimeters across and have spread to lymph nodes or other tissue near the breast.

V. Stage Four (IV) cancers are called metastasized cancers and have spread to other parts of the body. The seriousness of the diseases increases with each stage as the survival rates decrease.

Breast cancer is either noninvasive (confined to the site of origin) or invasive (spreading). Noninvasive breast cancers include ductal carcinoma in situ (also called intraductal carcinoma or DCIS) and lobular carcinoma in situ, or LCIS.

DCIS consists of cancer cells in the lining of the duct. DCIS is a non-invasive, early stage of cancer, but if left untreated, it may sometimes progress to an invasive, infiltrating ductal breast cancer. Although noninvasive, lobular carcinoma in situ is indicative of an increased risk of invasive cancer in both breasts.

Invasive cancer occurs when cancer cells spread beyond the basement membrane, which covers the underlying connective tissue in the breast. This tissue is rich in blood vessels and lymphatic channels that are capable of carrying cancer cells beyond the breast. Invasive breast cancers include infiltrating 
ductal carcinoma and infiltrating lobular carcinoma. Infiltrating ductal carcinoma is an invasive breast cancer that penetrates the wall of a duct. It comprises between $70 \%$ and $80 \%$ of all breast cancer cases. Infiltrating lobular carcinoma is an invasive cancer that has spread through the wall of a lobule. It accounts for between $10 \%$ and $15 \%$ of all breast cancers.

It may sometimes appear in both breasts and in several separate locations In recent years the occurrence of breast cancer has risen, but the death rate has fallen. Death rates did not drop significantly for women who did not have periodic screening mammograms. While new treatments and greater awareness of palpable lumps have improved a woman's chances for surviving breast cancer, screening mammography is considered an essential tool for early detection.

\section{MAMMOGRAPHY PROCESS}

Mammography is an X-ray technique for examining the breast. Like other radiographic examinations, an X-ray beam is passed through the tissue to record the variations in amounts of radiation that are absorbed. Since different tissues in the breast absorb different amounts of radiation, it is possible to distinguish features and details about the tissues being examined.

In screening mammography each breast is compressed into a relatively flat surface. Then an X-ray source on one side of the breast emits radiation through the breast. On the other side of the breast the radiation is recorded on film or by an electronic device. In a typical screening mammogram, each breast is examined from an overhead view, called craniocaudal, and from the side, called mediolateral.

The interior of the female breast contains mostly fatty and fibrous connective tissues. It is divided into about twenty sections called lobes, which are further subdivided into lobules. These are structures that contain milk-producing glands. These glands secrete milk into a system of ducts, which carry the milk through the breast and converge in a collecting chamber located just below the nipple.

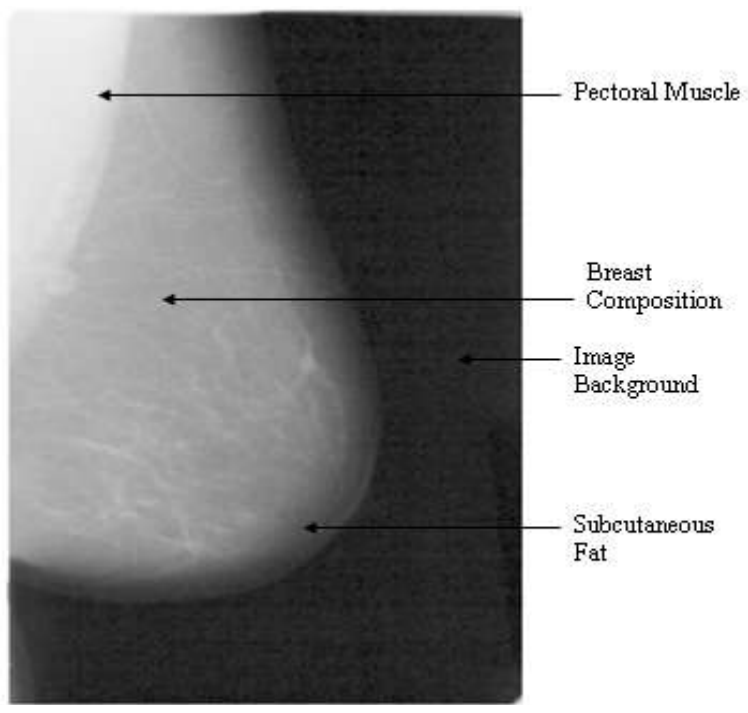

Figure 1. Labeled mammogram image
Figure 1 shows a mediolateral mammogram image in which the basic components of the breast are labelled.

Denser tissues in the breast display brighter intensities in the mammogram images. Muscles, fibroglandular tissue, malignant and benign masses, and vascular tissue appear brighter. Areas containing fat or skin appear darker.

Other important objects observed in mammograms are microcalcifications. These represent calcium deposits located in the breast tissue and are considered highly indicative of breast cancer. Microcalcifications appear as small, bright objects that stand out from the surrounding tissue. Figure 2 shows several examples of microcalcifications.

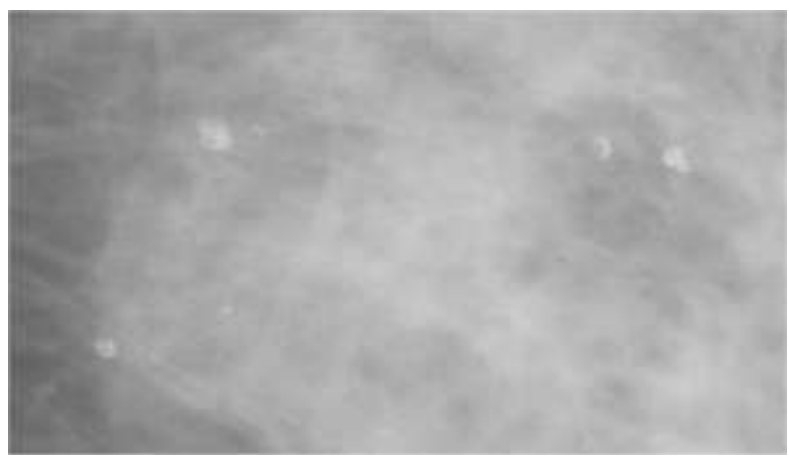

Figure 2. Microcalcification spots are shown

A category of abnormalities observed in mammogram images is architectural distortions. These are instances in which the normal structure of the breast is observably distorted. This includes spiculated areas and/or retraction from a focal point. These distortions are seen even though no mass is visible.

\section{MAMMOGRAM METHODS}

SEGMENTATION

A mass is space occupying lesion seen in at least two different projections defined with wide range of features that can indicate benign changes but can also be a part of malignant changes. Masses with round, smooth and circumscribed margins usually indicate benign changes while masses with spiculated, rough and blurry margins usually indicate a malignant mass. Some researchers have focused mainly on the detection of spiculated masses because of their high likelihood of malignancy.

The aim of the segmentation is to extract ROIs containing all masses and locate the suspicious mass candidates from the ROI. Segmentation of the suspicious regions on a mammographic image is designed to have a very high sensitivity and a large number of false positives are acceptable since they are expected to be removed in later stage of the algorithm [1]. Researchers have used several segmentation techniques and their combinations.

Global thresholding [3] is one of the common techniques for image segmentation. It is based on the global information, such as histogram. The fact that masses usually have greater intensity than the surrounding tissue can be used for finding global threshold value. On the histogram, the regions with an abnormality impose extra peaks while a healthy region has only a 
single peak [2]. After finding a threshold value the regions with abnormalities can be segmented.

Global thresholding is not a very good method to identify ROI because masses are often superimposed on the tissue of the same intensity level. Global thresholding has good results when used as a primary step of some other segmentation techniques.

Local thresholding is slightly better than global thresholding. The threshold value is defined locally for each pixel based on the intensity values of its neighbour pixels. Multiple pixels belonging to the same class (pixels at the periphery of the mass and pixels at the center of the mass) are not always homogenous and may be represented by different feature values. Li et al. [4] used local adaptive thresholding to segment mammographic image into parts belonging to same classes and an adaptive clustering to refine the results.

Markov random field (MRF) or Gibbs random field (GRF) is one of the segmentation methods in iterative pixel classification category. MRFs/GRFs are statistical methods and powerful modelling tools [5].

Székely et al. [6] used MRF in "fine" segmentation to improve the preliminary results provided by the "coarse" segmentation. In "coarse" segmentation the feature vector is calculated and passed to a set of decision trees that classifies the image segment.

After the "fine" segmentation they used a combination of three different segmentation methods: a modification of the radial gradient index method, the Bézier histogram method and dual binarization to segment a mass from the image.

Region growing and region clustering are also based on pixel classification. In region growing methods pixels are grouped into regions. A seed pixel is chosen as a starting point from which the region iteratively grows and aggregates with neighboring pixels that fulfill a certain homogeneity criterion.

Zheng et al. [7] used an adaptive topographic region growth algorithm to define initial boundary contour of the mass region and then applied an active contour algorithm to modify the final mass boundary contour.

Region clustering searches the region directly without initial seed pixel. Pappas [8] used a generalization of K-means clustering algorithm to separate the pixels into clusters based on their intensity and their relative location.

Li et al. [4] used an adaptive clustering to refine the result attained from the localized adaptive thresholding. Sahiner et al. [9] used K-means clustering algorithm followed by object selection to detect initial mass shape within the ROI. The ROI is extracted based on the location of the biopsied mass identified by a qualified radiologist. Initial mass shape detection is followed by an active contour segmentation method to refine the boundaries of the segmented mass.

Edge detection algorithms are based on the gray level discontinuities in the image. Basis for edge detection are gradients or derivatives that measure the rate of change in the gray level.
Rangayyan [10] described standard operators for edge detection such as Prewitt operator, Sobel operator, Roberts operator and Laplacian of Gaussian (LoG) operator.

Fauci et al. [11] developed an edge-based segmentation algorithm that uses iterative procedure, a ROI Hunter algorithm for selecting ROIs. ROI Hunter algorithm is based on the search of relative intensity maximum inside the square windows that form the mammographic image.

Petrick [12] used Laplacian of Gaussian filter in conjunction with density weighted contrast enhancement (DWCE). DWCE method enhances the structures within the mammographic image to make the edge detection algorithm able to detect the boundaries of the objects.

Zou et al. [13] proposed a method that uses gradient vector flow field (GVF) which is a parametric deformable contour model. After the enhancement of mammographic images with adaptive histogram equalization, the GVF field component with the larger entropy is used to generate the ROI.

Ferreira et al. [14] used active contour model (ACM) based on self-organizing network (SON) to segment the ROI. This model explores the principle of isomorphism and self-organization to create flexible contours that characterizes the shapes in the image. Yuan et al. [15] employed a dual-stage method to extract masses from the surrounding tissues.

Radial gradient index (RGI) based segmentation is used to yield an initial contour close to the lesion boundary location and a region-based active contour model is utilized to evolve the contour further to the lesion boundary.

\section{CONCLUSIONS}

Breast cancer is one of the major causes of death among women. Digital mammography screening programs can enable early detection and diagnose of the breast cancer which reduces the mortality and increases the chances of complete recovery. Screening programs produce a great amount of mammographic images which have to be interpreted by radiologists. Computeraided detection and diagnosis algorithms have been developed to help radiologists give an accurate diagnosis and to reduce the number of false positives. There are a lot of algorithms developed for detection of masses and calcifications. In this paper, methods that are commonly used were discussed.

\section{REFERENCES}

[1] Sampat, M.P., Markey, M.K., Bovik, A.C.: Computer-Aided Detection and Diagnosis in Mammography. In: Bovik, A.C. (ed.) Handbook of Image and Video Processing. Elsevier Academic Press, Amsterdam (2005)

[2] Cheng, H.D., Shi, X.J., Min, R., Hu, L.M., Cai, X.P., Du, H.N.: Approaches for Automated Detection and Classification of Masses in Mammograms. Pattern Recognition 39(4), 646-668 (2006)

[3] Brzakovic, D., Luo, X.M., Brzakovic, P.: An approach to automated detection of tumors in mammograms. IEEE Transactions on Medical Imaging 9(3), 233-241 (1990) 
[4] Li, H.D., Kallergi, M., Clarke, L.P., Jain, V.K., Clark, R.A. Markov Random Field for Tumor Detection in Digital Mammography. IEEE Transactions on Medical Imaging 14(3), 565-576 (1995)

[5] Li, L.H., Qian, W., Clarke, L.P., Clark, R.A., Thomas, J.: Improving Mass Detection by Adaptive and Multi-Scale Processing in Digitized Mammograms. Proceedings of SPIE-The International Society for Optical Engineering 3661 1, 490-498 (1999)

[6] Székely, N., Tóth, N., Pataki, B.: A Hybrid System for Detecting Masses in Mammographic Images. IEEE Transactions on Instrumentation and Measurement 55(3), 944-951 (2006)

[7] Zheng, B., Mello-Thoms, C., Wang, X.H., Gur, D.: Improvement of Visual Similarity of Similar Breast Masses Selected by Computer-Aided Diagnosis Schemes. In: 4th IEEE International Symposium on Biomedical Imaging: From Nano to Macro, ISBI 2007, April 12-15, pp. 516-519 (2007)

[8] Pappas, T.N.: An Adaptive Clustering Algorithm for Image Segmentation. IEEE Transactions on Signal Processing 40(4), 901-914 (1992)

[9] Sahiner, B., Hadjiiski, L.M., Chan, H.P., Paramagul, C., Nees, A., Helvie, M., Shi, J.: Concordance of ComputerExtracted Image Features with BI-RADS Descriptors for Mammographic Mass Margin. In: Giger, M.L., Karssemeijer, N. (eds.) Proc. of SPIE Medical Imaging 2008: Computer-Aided Diagnosis, vol. 6915 (2008)

[10] Rangayyan, R.M.: Biomedical Image Analysis. CRC Press LLC, Boca Raton (2005)
[11] Fauci, F., Bagnasco, S., Bellotti, R., Cascio, D., Cheran, S.C., De Carlo, F., De Nunzio, G., Fantacci, M.E., Forni, G., Lauria, A., Torres, E.L., Magro, R., Masala, G.L., Oliva, P., Quarta, M., Raso, G., Retico, A., Tangaro, S.: Mammogram Segmentation by Contour Searching and Massive Lesion Classification with Neural Network. In: 2004 IEEE Nuclear Science Symposium Conference Record, Rome, Italy, October 16-22, vol. 5, pp. 2695-2699 (2004)

[12] Petrick, N., Chan, H.P., Sahiner, B., Wei, D.: An Adaptive Density Weighted Contrast Enhancement Filter for Mammographic Breast Mass Detection. IEEE Transactions on Medical Imaging 15(1), 59-67 (1996)

[13] Zou, F., Zheng, Y., Zhou, Z., Agyepong, K.: Gradient Vector Flow Field and Mass Region Extraction in Digital Mammograms. In: 21st IEEE International Symposium on Computer-Based Medical Systems, CMBS 2008, Jyvaskyla, June 17-19, pp. 41- 43 (2008)

[14] Ferreira, A.A., Nascimento Jr., F., Tsang, I.R., Cavalcanti, G.D.C., Ludermir, T.B., de Aquino, R.R.B.: Analysis of Mammogram Using Self-Organizing Neural Networks Based on Spatial Isomorphism. In: Proceedings of International Joint Conference on Neural Networks, IJCNN 2007, Orlando, Florida, USA, August 12-17, pp. 1796-1801 (2007)

[15] Yuan, Y., Giger, M.L., Li, H., Sennett, C.: Correlative Feature Analysis of FFDM Images. In: Giger, M.L., Karssemeijer, N. (eds.) Proc. of SPIE Medical Imaging 2008: Computer-Aided Diagnosis, vol. 6915 (2008) 